\title{
Linkage of Decreased Bone Mass with Impaired Osteoblastogenesis in a Murine Model of Accelerated Senescence
}

\author{
Robert L. Jilka, Robert S. Weinstein, Kenshirou Takahashi, A. Michael Parfitt, and Stavros C. Manolagas \\ Division of Endocrinology and Metabolism, Center for Osteoporosis and Metabolic Bone Diseases, and Veterans Affairs Medical Center \\ GRECC, University of Arkansas for Medical Sciences, Little Rock, Arkansas 72205
}

\begin{abstract}
Bone marrow is the principal site for osteoclastogenesis and osteoblastogenesis; and an increase in the former has been linked with bone loss caused by acute loss of gonadal steroids. We have now used an established murine model of accelerated senescence and osteopenia (SAMP6) to test the hypothesis that reduced osteoblastogenesis is linked with decreased bone mass. At 1 mo of age, the number of osteoblast progenitors in SAMP6 marrow was indistinguishable from controls; however a threefold decrease was found at 3-4 mo of age. Impaired osteoblast formation was temporally associated with decreased bone formation and decreased bone mineral density, as determined by histomorphometric analysis of tetracycline-labeled cancellous bone and dualenergy x-ray absorptiometry, respectively. Osteoclastogenesis determined in ex vivo bone marrow cultures was also decreased in these mice, as was the number of osteoclasts in histologic sections. Moreover, unlike controls, senescenceaccelerated mice failed to increase osteoclast development after gonadectomy. The osteoclastogenesis defect was secondary to impaired osteoblast formation as evidenced by the fact that osteoclastogenesis could be restored by addition of osteoblastic cells from normal mice. These findings provide the first demonstration of a link between low bone mineral density and decreased osteoblastogenesis in the bone marrow and validate the senescence-accelerated mouse as a model of involutional osteopenia. (J. Clin. Invest. 1996. 97:1732-1740.) Key words: osteoporosis • aging • remodeling $\cdot$ bone formation $\bullet$ osteoclast formation
\end{abstract}

\section{Introduction}

The skeleton is continuously remodeled during adult life with the resorption of old bone by osteoclasts and its subsequent replacement by osteoblasts. This process is carried out in a highly coordinated fashion with a specific temporal and spatial pattern. Disturbances in the process result in an imbalance between resorption and formation and are responsible for most metabolic bone diseases including osteoporosis. Many of the clinical and epidemiological features of osteoporosis can be accounted for by a two-stage model of bone loss $(1,2)$. In this

Address correspondence to Robert L. Jilka, Ph.D., Division of Endocrinology and Metabolism, Slot 587, University of Arkansas for Medical Sciences, 4301 W. Markham St., Little Rock, AR 72205. Phone: 501-686-7896; FAX: 501-686-8148.

Received for publication 12 October 1995 and accepted in revised form 8 January 1996.

The Journal of Clinical Investigation

Volume 97, Number 7, April, 1996, 1732-1740 model, sex hormone deficiency results in an increase in both osteoclast and osteoblast activity with the former exceeding the latter, resulting in rapid bone loss. This phase is followed by a period of slow bone loss due to aging (3-9). It is generally agreed that the phase of rapid bone loss does not continue indefinitely, but its duration varies considerably among individuals. Confounding factors such as age-associated changes in vitamin D status or age-related development of subclinical hyperparathyroidism may result in continued elevated bone remodeling in some individuals (10-13). Nevertheless, the existence of an age-induced mechanism of bone loss, distinct from age-related alterations in hormone levels, is indicated by the inability of hormone replacement therapy to prevent bone loss indefinitely and the occurrence of bone loss with advancing age in sex hormone-replete men $(14,15)$.

A consistent histologic feature of the osteopenia that occurs during aging is a decrease in wall thickness, especially in trabecular bone $(16,17)$. Wall thickness is a measure of the amount of bone formed in a remodeling packet of cells and is determined by the number and activity of osteoblasts at the remodeling site. Considerable evidence suggests that the decline in wall thickness is due to the aging process itself, as opposed to hormone changes that occur with advancing age, such as alterations in the level of sex steroids or parathyroid hormone $(18,19)$. Hence, the osteopenia due to aging may be due to a deficit in the number of osteoblasts recruited to the remodeling site relative to the demand for them (17).

The bone-forming osteoblast and the bone-resorbing osteoclast originate from mesenchymal and hematopoietic progenitors of the marrow, respectively. The development of osteoblasts and osteoclasts are governed by locally produced cytokines and growth factors that influence the differentiation of osteoclasts from colony-forming unit-granulocyte/macrophage $(\mathrm{CFU}-\mathrm{GM})^{1}$ and/or the differentiation of osteoblasts from colony-forming unit-fibroblast (CFU-F). Based on extensive histomorphometric data from humans, as well as the results of experimentation in animal models, we recently proposed the hypothesis that inappropriate production of osteoclasts or inadequate production of osteoblasts, as opposed to changes in the activity of these cells, is responsible for the mismatch between formation and resorption of bone in postmenopausal and age-related osteopenia, respectively (20).

To examine the relationship between osteoblast progenitor development in the bone marrow, bone remodeling, and bone mass, we have used the SAMP6 mouse, a model of early senes-

1. Abbreviations used in this paper: $\mathrm{BMC}$, bone mineral content; $\mathrm{BMD}$, bone mineral density; CFU-F, colony-forming unit-fibroblast; CFU-GM, colony-forming unit-granulocyte/macrophage; CFU-OB, colony-forming unit-osteoblast; DEXA, dual-energy x-ray absorptiometry; LDH, lactate dehydrogenase; TRAPase, tartrate-resistant acid phosphatase. 
cence that exhibits osteopenia $(21,22)$. The SAMP6 strain as well as the "normal" control strain SAMR1 were developed over the past $20 \mathrm{yr}$ by investigators at Kyoto University by brother-sister mating of $\mathrm{AKR} / \mathrm{J}$ mice for $>50$ generations. When reared under specific pathogen-free conditions, SAMP6 exhibit normal incidence of tumors and other specific abnormalities. Thus, the confounding effects of age-related diseases of inbred mice do not account for either the rapid aging or the osteopenia of SAMP6 mice. The osteopenic phenotype of this strain may have arisen from unfavorable combinations of alleles at multiple loci of the genome affecting bone mass. Alternatively, retroviruses from the AKR/J parental strain, or another aberrantly introduced strain, may have become activated when placed in a heterologous genetic background after a presumed (accidental) outcrossing at the initial stages of the development of this strain (23).

In this report we present evidence for a tight linkage among decreased osteoblastogenesis in the bone marrow, diminished bone formation in remodeling cancellous bone, and low bone mineral density (BMD).

\section{Methods}

Animals. SAMP6 mice and SAMR1 mice were obtained from a colony established from breeders kindly provided to us by Dr. Toshio Takeda of Kyoto University (Kyoto, Japan). Individual animals were electronically tagged (BioMedic Data System Inc., Maywood, NJ) at weaning. For some experiments, 3-4-mo-old mice were gonadectomized as described previously $(24,25)$. Neonatal C57Bl/6J mice were obtained from Harlan Sprague Dawley, Inc. (Indianapolis, IN). All animals were maintained and used in accordance with National Institutes of Health guidelines on the care and use of laboratory animals.

Detection and quantification of osteoblast progenitors. CFU-F were detected by the formation of a colony of adherent fibroblastoid cells in ex vivo marrow cell cultures (26). The presence of osteoblasts in these colonies was assayed by determining the ability of cells present in the colony to form a mineralized matrix (27). Mineralizing colonies were designated as colony-forming unit-osteoblast (CFU$\mathrm{OB})$. The number of CFU-F and CFU-OB in murine bone marrow was determined by limiting dilution assay (27). Marrow cells were obtained from the femur (24), and replicate cultures $(n=16-24)$ of serially diluted cells were established from each animal in $0.3-\mathrm{cm}^{2}$ wells of a 96-well plate at 6-8 seeding densities that ranged from $\sim 300,000$ to 10,000 cells per well. Cells were cultured in phenol red-free $\alpha$ MEM containing $15 \%$ preselected FBS, $50 \mu \mathrm{M}$ ascorbic acid, and 10 $\mathrm{mM} \beta$-glycerophosphate. One-half of the medium was replaced every $5 \mathrm{~d}$. For the determination of CFU-F, cultures were maintained for $10 \mathrm{~d}$, fixed with neutral buffered formalin, stained for alkaline phosphatase (kit 180; Sigma Immunochemicals, St. Louis, MO), and counterstained with hematoxylin. Colonies containing $>50$ cells were designated as CFU-F colonies. For the determination of CFU-OB, cells were cultured for $25 \mathrm{~d}$, fixed in $50 \%$ ethanol, $18 \%$ formaldehyde, and stained for calcium deposits using either Von Kossa stain or alizarin red. The percentage of wells that contained CFU-F or CFU-OB was determined for each cell concentration and the number of progenitors per $10^{6}$ marrow cells was graphically determined as described previously (27).

Analysis of individual CFU-F. Marrow cells were seeded in a 96well plate at 10,000, 20,000 or 30,000 per well $(n=48$ wells for each seeding density) and cultured as described above for the determination of CFU-F. Cells were dried and then fixed with neutral buffered formalin $(10 \%)$ at $4^{\circ} \mathrm{C}$ for $10 \mathrm{~min}$. The activity of lactate dehydrogenase (LDH) was used as an index of the number of cells present in each well and was determined by coupling the enzyme reaction to the reduction of a tetrazolium salt to a formazan as described previously
(28). Each well contained $100 \mu \mathrm{l}$ of substrate solution consisting of 0.2 M Tris buffer, $\mathrm{pH} 8.2,27 \mathrm{mM}$ sodium $\mathrm{L}(+)$-lactate, $0.65 \mathrm{mM} \beta$-nicotinamide adenine dinucleotide, $1.3 \mathrm{U} / \mathrm{ml}$ diaphorase, and $0.3 \mathrm{mg} / \mathrm{ml}$ 3-(4,5-dimethylthiazol-2-yl)-5-(3-carboxymethoxyphenyl)-2-(4-sulfophenyl)-2H-tetrazolium, inner salt (MTS). The rate of MTS reduction was determined by measuring the absorbance at $490 \mathrm{~nm}$ over a 12-16-h period, using a microtiter plate reader. LDH activity was expressed as the change in $\mathrm{OD}^{490}$ per minute. Preliminary experiments indicated that as few as $50 \mathrm{MC} 3 \mathrm{~T} 3-\mathrm{E} 1$ osteoblastic cells could reliably be detected using this assay (data not shown). After determination of LDH activity, wells were rinsed with distilled water, and the alkaline phosphatase activity in each well was quantified as follows. Each well contained $100 \mu \mathrm{l}$ of $0.1 \mathrm{M}$ 2-amino-2-methyl-1-propanol buffer, $\mathrm{pH}$ 10.4, and $7 \mathrm{mM} p$-nitrophenyl phosphate (kit 104; Sigma Immunochemicals). The production of $p$-nitrophenol was determined by measuring the absorbance at $402 \mathrm{~nm}$ over a $12-16-\mathrm{h}$ period. One microunit of activity was defined as that amount of enzyme capable of hydrolyzing 1 pmol $p$-nitrophenylphosphate per minute at room temperature. Data on cell number (LDH activity) and alkaline phosphatase content were analyzed only in the case of wells that contained a single colony, determined by microscopic examination.

Bone histomorphometry. Mice were injected (intraperitoneally) with tetracycline hydrochloride $(15 \mathrm{mg} / \mathrm{kg}$ body weight $)$ at 8 and $3 \mathrm{~d}$ before killing. The distal femur and lumbar vertebrae were fixed at $4^{\circ} \mathrm{C}$ in phosphate-buffered $10 \%$ formalin, $\mathrm{pH} 7.4$, containing $0.5 \%$ sucrose, and embedded in methyl methacrylate as described previously $(29,30)$. Uniform positioning of embedded bones was assured by placing them into a carved groove in a preformed, sticky methacrylate wafer at the bottom of each embedding vial. 5- $\mu$ m-thick longitudinal sections taken from halfway through the specimens were cut on Zeiss 350 heavy-duty microtome (Carl Zeiss, Inc., Thornwood, NY) with a D profile knife (Delaware Diamond Knives Corp., Wilmington, DE). Osteoid and osteoblasts were best distinguished when stained with $0.25-1 \%$ toluidine blue, $\mathrm{pH} 2.8-3.2$. Murine osteoclasts were identified by staining for tartrate-resistant acid phosphatase (TRAPase) (Sigma Immunochemicals). Adjacent 8- $\mu$ m-thick sections were left unstained for epifluorescence observations.

Static and dynamic histomorphometric measurements were made as described previously using a computer and digitizer tablet (OsteoMeasure; Osteometrics, Inc., Atlanta, GA) interfaced to a Zeiss Axioscope (Carl Zeiss, Inc.) with a drawing tube attachment $(29,30)$. The tetracycline labels were measured at a magnification of 400 (numerical aperture 0.75). The terminology used is that recommended by the Histomorphometry Nomenclature Committee of the American Society for Bone and Mineral Research (31). To ensure that only remodeling bone of the secondary spongiosa was examined, measurements were restricted to the area between 1 and $5 \mathrm{~mm}$ distal to the growth plate-metaphyseal junction of the distal femur, and the central cancellous bone of the vertebrae. We avoided areas of bone undergoing modeling, specifically the endocortical margins of the diaphysis, and the cancellous bone furthest away from the growth plate. To estimate bone turnover, the rate of bone formation per unit of bone area was calculated as the bone formation rate per bone perimeter multiplied by the perimeter to area ratio, and expressed as the percentage of bone area remodeled per day. Values are expressed as means \pm SD.

Bone densitometry. Dual-energy x-ray absorptiometry (DEXA) was performed on a QDR-2000 Plus densitometer (Hologic, Waltham, MA) that was calibrated daily with a hydroxyapatite phantom of the human lumbar spine. Each set of animal scans was preceded by scanning a plastic embedded murine phantom. Before scanning, animals were sedated (intramuscularly) with $120 \mu \mathrm{g} / 10$ grams body weight sodium pentobarbital (Sigma Immunochemicals). Scans were done with the animals positioned prone. Measurements were made with a customized mouse whole body software package (Hologic) that allowed specified region of interest windows and subtraction of the animal identification transponder device. The global window was defined as the whole body image minus the calvarium, mandible, and teeth 
$(183 \times 138$ pixels $)$. The spine window was a rectangle 23 pixels wide and $\sim 80$ pixels long (depending on animal body length), reaching from just below the skull to the base of the tail. The hindquarters windows were left and right boxes $(47 \times 81$ pixels $)$ that enclosed the femora, tibiofibulare, and feet. The scans were done with a $1.270-\mathrm{mm}$ diameter collimator, $0.762-\mathrm{mm}$ line spacing, $0.380-\mathrm{mm}$ point resolution, and an acquisition time of $9 \mathrm{~min}$. Values were expressed as BMD in $\mathrm{mg} / \mathrm{cm}^{2}$.

Duplicate BMD determinations with repositioning between the scans $(n=32$ mice $)$ showed that the mean difference in BMD between the two measurements was not significantly different from zero, thus proving that repositioning did not influence the measurement. The mean coefficients of variation for the short-term reproducibility studies were $2.17 \%$ for the global measurement, $2.19 \%$ for the spine, and $3.10 \%$ for the hindquarters. Long-term reproducibility was assessed by repeated measurement of the murine phantom. In a 6-mo period, 55 measurements were made with a coefficient of variation of $4.39 \%$.

The accuracy of DEXA measurements was demonstrated by determining bone mineral content (BMC) and ash weight on the same murine bone samples as follows. Mice $(n=47)$ that were $23-378 \mathrm{~d}$ old and weighing 9.0-69.8 grams were killed by $\mathrm{CO}_{2}$ inhalation. After determination of BMC by DEXA, the entire skeleton (excluding skull and tail) was harvested for ashing. In 24 of these animals, the spine, hindquarters, and remaining bones were each ashed separately. Ashing was done in a muffle furnace at $800^{\circ} \mathrm{C}$ for $24 \mathrm{~h}$ in covered quartz crucibles (Fisher Scientific, Pittsburgh, PA). After determining ash weight, data were analyzed by linear regression with ash weight as the independent variable and BMC as the dependent variable (Fig. 1). We observed a strong linear relationship between ash weight and global BMC $(r=0.96, P<0.00001)$. The relationship between the ash weight and BMC of the spine and hindquarter subregions was also strong $(r=0.97$ and 0.95 , respectively, $P<0.00001)$. For the three relationships, the slope ranged from 0.928 to 1.194 and the intercept from -0.127 to 0.048 . The relationships were not affected by sex, weight, or age of the mice.

Assay of osteoclast formation. Marrow cells (four to six replicate cultures) from each animal were cultured separately at $1.5 \times 10^{6}$ per $2-\mathrm{cm}^{2}$ well and maintained for $9 \mathrm{~d}$ in the presence of $10^{-8} \mathrm{M}$ $1,25(\mathrm{OH})_{2} \mathrm{D}_{3}$ and $10 \%$ FBS in $\alpha \mathrm{MEM}$ as described previously $(24,25$, 32 ). We also used a coculture assay to quantify osteoclast progenitors (24). In these experiments, exogenous stromal/osteoblastic cells from neonatal murine calvaria cells were provided as support for osteoclast progenitors. Stromal/osteoblastic cells were prepared by sequential collagenase digestion of calvaria dissected from 3-5-d-old C57Bl/6 J mice as described previously $(24,32,33)$. Marrow cells $(200,000$ per $2-\mathrm{cm}^{2}$ well) were cocultured with 40,000 calvaria cells and maintained for $8 \mathrm{~d}$ in the presence of $10 \mathrm{nM} 1,25(\mathrm{OH})_{2} \mathrm{D}_{3}$ and $10 \%$ FBS in $\alpha M E M$. The number of osteoclasts formed was determined by counting cells that both bound ${ }^{125} \mathrm{I}$-calcitonin and expressed TRAPase. The number of osteoclasts formed per femur was calculated using the marrow cell yield from the animal.

$C F U-G M$ assay. Marrow cells were cultured separately to establish three replicate cultures. Cells were plated at 100,000 cells per $10-\mathrm{cm}^{2}$ culture dish containing $1.5 \mathrm{ml}$ of semisolid methylcellulose medium with pokeweed mitogen-stimulated spleen cell conditioned medium (No. M3430; Stem Cell Technologies, Inc., Vancouver, British Columbia, Canada). After $7 \mathrm{~d}$, CFU-GM colonies ( $>50$ cells) were enumerated. The number of CFU-GM formed per femur was calculated using the marrow cell yield from the animal.

Assessment of individual CFU-F colonies for ability to support osteoclast formation. Marrow cells were seeded at 20,000 per well in each well of a 96-well plate. After $5 \mathrm{~d}$ of culture spleen cells from normal Swiss/Webster mice were added as a source of osteoclast progen-

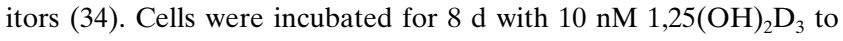
stimulate osteoclast formation. At the end of the experiment, each well was examined microscopically for the presence of CFU-F colonies and osteoclastic cells, identified by staining for TRAPase.

Statistics. Student's $t$ test was used to test for significant differences between means of two groups, after testing for equivalence of variances and normal distribution of data. Data which did not meet this criteria were analyzed by the Mann-Whitney rank sum test. Correlation coefficients were calculated to test for an association between two independently measured variables. Fisher's exact test was used to test for significant differences in the proportion of positive elements in two populations.

\section{Results}

Analysis of osteoblast progenitors of the bone marrow. Growing SAMR1 and SAMP6 mice ( $\sim 1$ mo old) had identical numbers of CFU-F and CFU-OB in the bone marrow (Fig. 2 $A$ ). On the other hand, marrow cells from 3-4-mo-old adult SAMP6 mice contained approximately threefold fewer CFUOB than SAMR1 (Fig. 2 B); but the number of CFU-F present in the marrow of SAMP6 and SAMR1 mice remained identical. Analysis of individual CFU-F colonies of 3-mo-old mice revealed that a larger proportion of the SAMP6 colonies was smaller and contained less alkaline phosphatase than colonies of SAMR1 mice (Fig. 3, $A$ and $B$ ). The median cell number index for SAMR1 colonies was $0.44 \times 10^{-3} \mathrm{OD} / \mathrm{min}$ vs. $0.11 \times$ $10^{-3} \mathrm{OD} / \mathrm{min}$ in the case of SAMP6 colonies $(P<0.01$ by Mann-Whitney rank sum test). The median alkaline phos-
A global

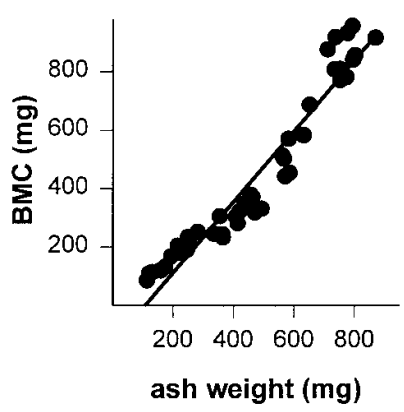

B spine

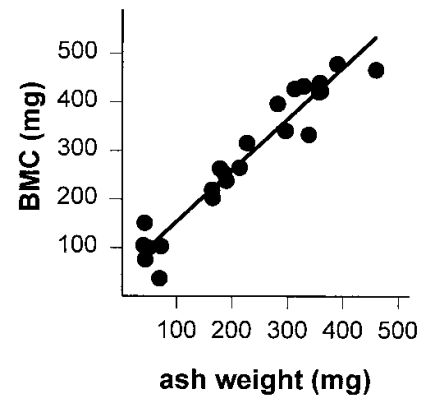

C hindquarters

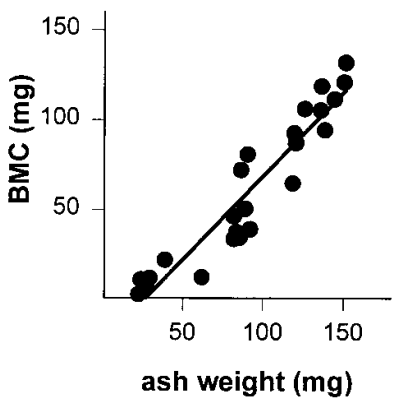

Figure 1. Correlation of BMD with ash weight. The global, spine, and hindquarters BMD were determined in 71 mice, weighing between 15 and 50 grams as described in Methods. The mice were then killed and bones dissected for ashing. In 47 of these animals, the entire skeleton (minus the head and tail) was ashed $(A)$. In 24 of these animals, the spine $(B)$ and hindquarters $(C)$ were ashed separately. The best fit line shown was determined by linear regression with ash weight as the independent variable and the DEXA-derived BMC as the dependent variable. 


\section{A 3-4 weeks of age}
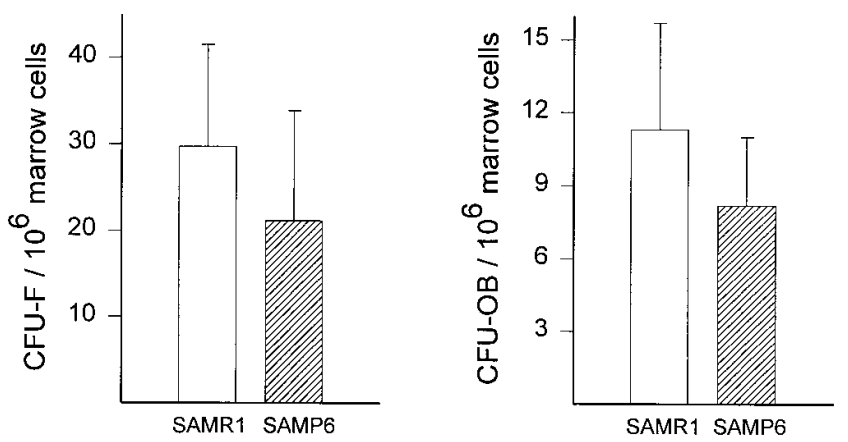

B 3-4 months of age
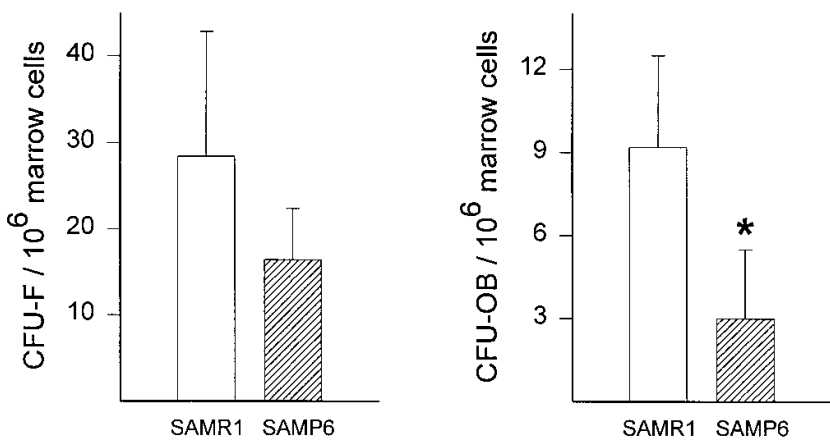

Figure 2. Quantification of CFU-F and CFU-OB by limiting dilution assay. Marrow cells were obtained from the femurs of SAMR1 and SAMP6 mice at 3-4 wk of age $(A)$ and at 3-4 mo of age $(B)$. Both males and females were used. Cells from each mouse were cultured separately as described in Methods for the determination of CFU-F and $\mathrm{CFU}-\mathrm{OB}$ by limiting dilution assay. Bars represent the mean $( \pm \mathrm{SD})$ number of progenitors per $10^{6}$ marrow cells $(n=5-8$ per group). $* P<0.05$ vs. SAMR1 mice. There was no difference in osteoblast progenitors between males and females of the same strain.

phatase activity of SAMR1 colonies was 2.49 vs. $0.96 \mu \mathrm{U} / \mathrm{col}-$ ony for SAMP6 colonies $(P<0.01)$. However, when calculated on a per cell basis, the level of alkaline phosphatase expression was identical in CFU-F colonies from the two mouse strains, regardless of colony size (data not shown). Thus, marrow of SAMP6 mice exhibited diminished numbers of progenitors capable of giving rise to large colonies with high levels of alkaline phosphatase.

Bone histomorphometry. The cancellous bone of the distal femur and lumbar vertebra displayed numerous resorption and formation surfaces (Fig. 4). Cement lines or reversal lines, which are deposited after the osteoclasts resorb bone, demarcate the old bone from the new. The scalloped appearance of the reversal line reflects previous episodes of osteoclast action at the remodeling site. We used TRAPase staining to identify osteoclasts because we found that murine osteoclasts were difficult to recognize morphologically due to their flattened appearance, shallow Howship's lacunae, and the presence of only one to three nuclei (Fig. $4 \mathrm{~B}$ ). Static and dynamic histomorphometric measurements in 3-mo-old adult SAMR1 and SAMP6 mice are shown in Table I. Cancellous bone area was significantly decreased in the lumbar vertebrae of SAMP6 mice, compared with that of SAMR1. The decreased bone area was characterized by a significant increase in the mean distance between individual trabeculae and a decrease in trabecular number. Trabecular width, osteocyte number, and the osteoid area, perimeter, and width were similar in SAMR1 and SAMP6 vertebral bone. The number of osteoblasts was highly variable when expressed as a percentage of the cancellous bone perimeter. However, there was a significant reduction in the number of osteoblasts in SAMP6 when expressed per unit area of cancellous tissue. The number of osteoclasts was dramatically decreased in SAMP6, whether expressed as a percentage of the cancellous perimeter, as the number of osteoclasts per millimeter of cancellous perimeter, or per unit area of cancellous tissue. Vertebral cancellous bone of SAMP6 mice showed a decreased tetracycline-labeled mineralizing perimeter compared with SAMR1. Furthermore, there was a significant reduction in the rate of mineral apposition and bone formation in SAMP6 mice. Bone formation rate expressed per bone area, an estimate of bone turnover (percentage per day), tended to be decreased in SAMP6 mice as compared with SAMR1 $(P=0.2)$.

In the distal femur, the cancellous bone area, trabecular spacing, trabecular number, and osteocyte number were similar in the two strains. However, there was a significant reduction in the trabecular width in distal femur of SAMP6 mice. Osteoid area, perimeter, and width were similar in SAMR1 and SAMP6 femoral bone. In SAMP6 mice, there was a significant reduction in the number of osteoblasts per unit area of cancellous tissue and in the number of osteoclasts per millimeter of cancellous perimeter, compared with the controls. In addition, the mineralizing perimeter and bone formation rate were reduced. As noted in the vertebrae, there was a trend toward decreased bone turnover in the distal femur of SAMP6 $(P=0.07)$.

Concurrent determination of CFU-OB, by limiting dilution assay, was also performed on marrow cells from the mice used for histomorphometric studies shown in Table I. The number of CFU-OB was directly correlated with the vertebral mineral appositional rate $(r=0.91, P=0.032)$, to the vertebral cancellous bone area $(r=0.88, P=0.047)$, to the femoral mineralizing perimeter $(r=0.88, P=0.047)$, and to the femoral osteoclast perimeter $(r=0.88, P=0.047)$.

The bone formation rates in the lumbar vertebrae of 3-4-wkold SAMR1 and SAMP6 mice were not significantly different $\left(0.469 \pm 0.035\right.$ vs. $0.587 \pm 0.149 \mu \mathrm{m}^{3} / \mu \mathrm{m}^{2} / \mathrm{d}$, respectively). In the lumbar vertebrae of 15-mo-old SAMR1 mice, tetracycline labels were rare, but one double label and several single labels were noted. In contrast, tetracycline-labeled cancellous perimeter was absent from the bone of 15-mo-old SAMP6 mice.

Bone densitometry. The global, spine, and hindquarters BMD were measured in weaned growing animals (36-38 d old), in sexually mature young adults (93-128 d old) and in very old animals (436-486 d old). As shown in the data of Table II, the BMD of the three skeletal sites in the 36-38-d-old animals was similar in SAMP6 and SAMR1 mice. In 3-4-mo-old adults, there was a significant decrease in global and spine BMD of SAMP6 mice; but there was no significant difference in the hindquarters BMD. Very old SAMP6 mice showed significant decreases in all three skeletal sites when compared with very old SAMR1 mice or 3-4-mo-old SAMP6 mice. However, the BMD in the very old SAMR1 mice was not significantly different from that seen in 3-4-mo-old SAMR1 mice. 


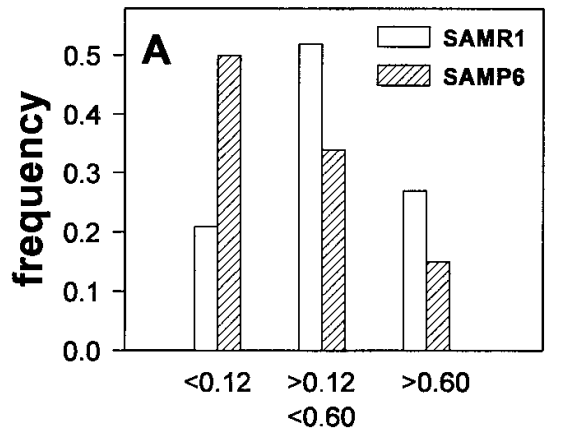

cell number (OD /min)

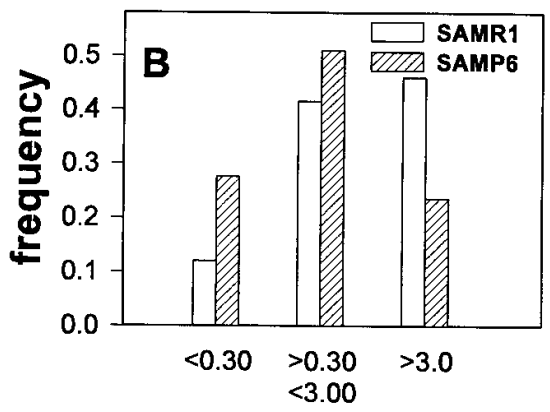

alkaline phosphatase $(\mu \mathrm{U} /$ colony)
Figure 3. Determination of cell number and alkaline phosphatase in individual CFU-F colonies of SAMR1 and SAMP6 mice. Pooled marrow cells from 3-mo-old female SAMR1 or SAMP6 mice were cultured in 96-well plates for the determination of cell number and alkaline phosphatase activity of individual CFU-F colonies as described in Methods. 49 colonies from SAMR1 and 26 colonies from SAMP6 were analyzed. Data shown represent the fraction of the colonies that exhibits the indicated range of cell number index expressed as OD $^{490}$ units/min $\left(\times 10^{-3}\right)(A)$ or the indicated range of alkaline phosphatase activity $(B)$. Statistical analysis by the Mann-Whitney rank sum test indicated a significant decrease in cell number index, as well as alkaline phosphatase activity, in colonies of SAMP6 mice as compared with that of SAMR1 mice $(P<0.01)$.
Osteoclastogenesis. In view of the evidence that osteoblastogenesis is defective in marrow of adult SAMP6 mice, we examined the possibility that the marrow of SAMP6 mice was also deficient in the stromal/osteoblastic cells that are required for osteoclast formation. Marrow cells from SAMP6 mice formed significantly fewer osteoclasts than cells from SAMR1 mice (Fig. $5 \mathrm{~A}$ ). This finding could be due to a decrease in the number of osteoclast progenitors and/or a decrease in stromal/
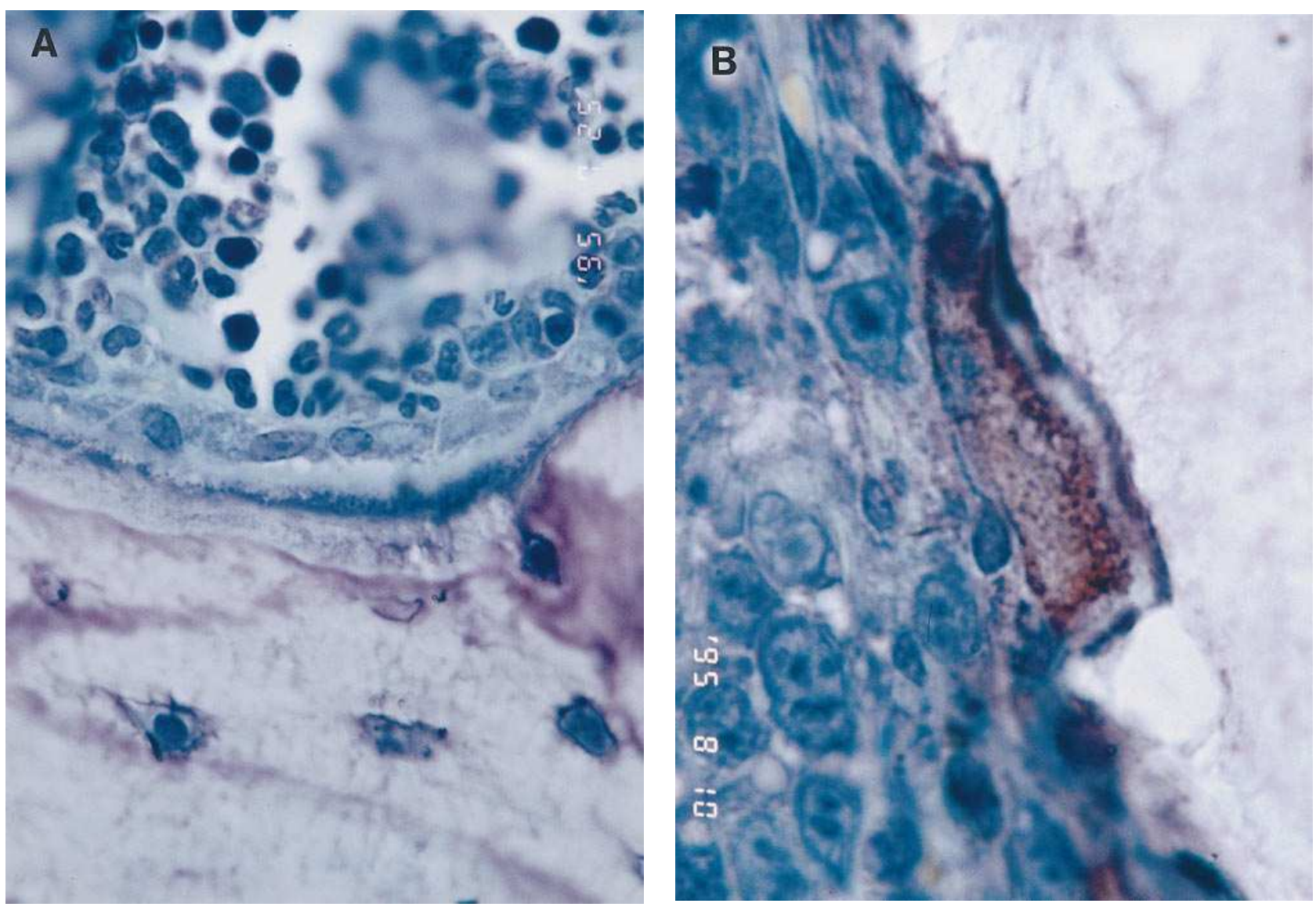

Figure 4. Photomicrographs of murine cancellous bone. In A, osteoid is light blue and mineralized bone is light purple. Lining the osteoid seam are a row of plump osteoblasts with perinuclear halos typical of their prominent Golgi apparatus. Large dark blue staining osteocytes are present deep within the mineralized bone. Dark purple-stained reversal lines mark the extent of previous episodes of bone remodeling. Toluidine blue stain, original magnification $\times 400$. In $B$, a TRAPase-positive osteoclast is present in a shallow resorption cavity. The nuclei are polarized away from the bone and the red TRAPase granules are adjacent to the clear zone or interface between the osteoclast and the mineralized bone perimeter. Photomicrographs are from cancellous bone of the distal femur of a 3-mo-old SAMR1 mouse. Toluidine blue/TRAPase stain, original magnification $\times 1,600$. 
Table I. Cancellous Bone Histomorphometry in 3-mo-old SAMR1 and SAMP6 Mice

\begin{tabular}{|c|c|c|c|c|}
\hline & \multicolumn{2}{|c|}{ Lumbar vertebrae } & \multicolumn{2}{|c|}{ Distal femur } \\
\hline & SAMR1 & SAMP6 & SAMR1 & SAMP6 \\
\hline Cancellous bone area $(\%)$ & $22.90 \pm 6.31$ & $8.57 \pm 0.92 *$ & $13.35 \pm 4.61$ & $9.29 \pm 0.94$ \\
\hline Trabecular number (per millimeter) & $5.72 \pm 1.17$ & $2.87 \pm 0.38 *$ & $2.95 \pm 0.97$ & $2.57 \pm 0.33$ \\
\hline Trabecular spacing $(\mu \mathrm{m})$ & $164 \pm 35$ & $359 \pm 28^{*}$ & $316 \pm 116$ & $337 \pm 36$ \\
\hline Trabecular width $(\mu \mathrm{m})$ & $39.7 \pm 5.2$ & $30.5 \pm 7.3$ & $45.0 \pm 1.4$ & $36.2 \pm 1.4 *$ \\
\hline Osteoid area $(\%)$ & $2.2 \pm 0.6$ & $1.4 \pm 1.0$ & $2.76 \pm 0.42$ & $3.34 \pm 1.38$ \\
\hline Osteoid width $(\mu \mathrm{m})$ & $3.3 \pm 0.4$ & $2.4 \pm 0.6$ & $4.4 \pm 1.0$ & $4.3 \pm 0.1$ \\
\hline Osteoblast perimeter (\%) & $5.9 \pm 3.3$ & $4.7 \pm 3.3$ & $6.5 \pm 1.9$ & $5.8 \pm 4.3$ \\
\hline Number of osteoblasts per millimeter & $4.9 \pm 1.9$ & $3.1 \pm 2.0$ & $7.1 \pm 3.6$ & $4.5 \pm 2.5$ \\
\hline Number of osteoblasts per square millimeter & $47.9 \pm 22.5$ & $15.2 \pm 9.1^{*}$ & $44.1 \pm 29.7$ & $23.0 \pm 9.7 *$ \\
\hline Number of osteocytes per bone area & $8.7 \pm 3.8$ & $6.7 \pm 2.9$ & $16.4 \pm 2.1$ & $13.6 \pm 5.0$ \\
\hline Osteoclast perimeter $(\%)$ & $4.76 \pm 2.37$ & $0.35 \pm 0.42 *$ & $1.36 \pm 1.05$ & $0.06 \pm 0.10^{*}$ \\
\hline Number of osteoclasts per millimeter & $2.24 \pm 1.01$ & $0.28 \pm 0.27 *$ & $0.48 \pm 0.40$ & $0.02 \pm 0.03^{*}$ \\
\hline Number of osteoclasts per square millimeter & $20.66 \pm 6.52$ & $1.37 \pm 1.28 *$ & $2.80 \pm 2.78$ & $0.12 \pm 0.20^{*}$ \\
\hline Mineralizing perimeter $(\%)$ & $6.97 \pm 5.91$ & $2.09 \pm 1.24 *$ & $4.79 \pm 0.45$ & $1.96 \pm 1.45^{*}$ \\
\hline Mineral appositional rate $(\mu \mathrm{m} / \mathrm{d})$ & $1.20 \pm 0.32$ & $0.46 \pm 0.11 *$ & $1.91 \pm 0.46$ & $1.53 \pm 0.21$ \\
\hline Bone formation rate per bone perimeter $\left(\mu \mathrm{m}^{3} / \mu \mathrm{m}^{2} / \mathrm{d}\right)$ & $0.095 \pm 0.102$ & $0.009 \pm 0.005 *$ & $0.092 \pm 0.028$ & $0.029 \pm 0.023^{*}$ \\
\hline Bone formation rate per bone area $(\% / d)$ & $0.377 \pm 0.366$ & $0.052 \pm 0.018$ & $0.349 \pm 0.132$ & $0.130 \pm 0.082$ \\
\hline
\end{tabular}

$* P<0.05$ vs. SAMR1.

osteoblastic support for osteoclast formation in ex vivo marrow cell cultures from SAMP6 mice. However, the number of CFU-GM was identical in marrow of SAMR1 and SAMP6 mice as determined in an ex vivo colony assay (Fig. 5 B). Furthermore, osteoclast formation in ex vivo cultures of marrow cells from SAMP6 mice was slightly higher than SAMR1 mice when marrow cells were cultured together with exogenous stromal/osteoblastic cells prepared from calvaria of normal neonatal $\mathrm{C} 57 \mathrm{Bl} / 6 \mathrm{~J}$ mice (Fig. $5 \mathrm{C}$ ). In these cocultures, there was a four- to eightfold increase in the number of osteoclasts, compared with those seen in marrow cells cultured alone.

To directly establish whether marrow of SAMP6 mice had reduced numbers of osteoclastogenic support cells, we compared osteoclast formation in CFU-F colonies of SAMP6 or SAMR1 mice that were cocultured with spleen cells as a source of osteoclast progenitors, as described in Methods. We found that whereas $40 \%$ of the CFU-F colonies (total $n=60$ ) from SAMR1 mice contained osteoclast-like cells, only $14 \%$ of the CFU-F colonies (total $n=35$ ) derived from SAMP6 mice contained osteoclast-like cells. Thus, significantly fewer CFU-F colonies of SAMP6 mice contained stromal/osteoblastic cells capable of supporting osteoclast formation $(P<0.02$ by Fisher's exact test).

We and others have shown previously that the stromal/osteoblastic cells of the marrow are a target of the action of sex steroids to suppress IL- 6 production and that IL-6 mediates the increase in osteoclast formation and the bone loss caused by loss of sex steroids $(6,24,25,32)$. In view of the evidence that marrow of SAMP6 mice is deficient in cells of the osteoblast lineage, we studied the effect of loss of sex steroids on osteoclast progenitors in these mice. As shown in Fig. 6, loss of estrogens or androgens had no effect on osteoclast progenitors of the marrow of SAMP6 mice, regardless of whether osteoclast formation was assessed in marrow cells cultured alone or in marrow cells cocultured with stromal/osteoblastic cells from normal neonatal mice. Nevertheless, sex steroid deficiency caused the expected increase in osteoclast progenitors in SAMR1 mice.

Table II. DEXA Determination of BMD

\begin{tabular}{|c|c|c|c|c|c|c|c|c|}
\hline \multirow[b]{3}{*}{ Age (d) } & \multirow{2}{*}{\multicolumn{2}{|c|}{ Number analyzed }} & \multicolumn{6}{|c|}{$\operatorname{BMD}\left(\mathrm{mg} / \mathrm{cm}^{2}\right)$} \\
\hline & & & \multicolumn{2}{|c|}{ Global } & \multicolumn{2}{|c|}{ Spine } & \multicolumn{2}{|c|}{ Hindquarters } \\
\hline & SAMR1 & SAMP6 & SAMR1 & SAMP6 & SAMR1 & SAMP6 & SAMR1 & SAMP6 \\
\hline $33-38$ & 13 & 15 & $37.5 \pm 2.6$ & $33.6 \pm 3.2$ & $41.5 \pm 3.3$ & $38.0 \pm 3.6$ & $35.5 \pm 3.6$ & $29.6 \pm 5.3$ \\
\hline $91-149$ & 46 & 47 & $50.6 \pm 3.2$ & $48.0 \pm 1.5$ & $56.9 \pm 3.3$ & $53.1 \pm 1.9 *$ & $53.8 \pm 4.7$ & $52.9 \pm 3.8$ \\
\hline $463-486$ & 6 & 6 & $51.7 \pm 4.6$ & $39.2 \pm 2.0^{* \neq}$ & $57.9 \pm 6.3$ & $44.0 \pm 2.9^{* \ddagger}$ & $56.4 \pm 2.7$ & $40.0 \pm 2.8^{* \ddagger}$ \\
\hline
\end{tabular}

Data shown are the mean \pm SD. $* P<0.05$ vs. SAMR1 of the same age; ${ }^{*} P<0.05$ vs. $91-149$-d-old SAMP6. 

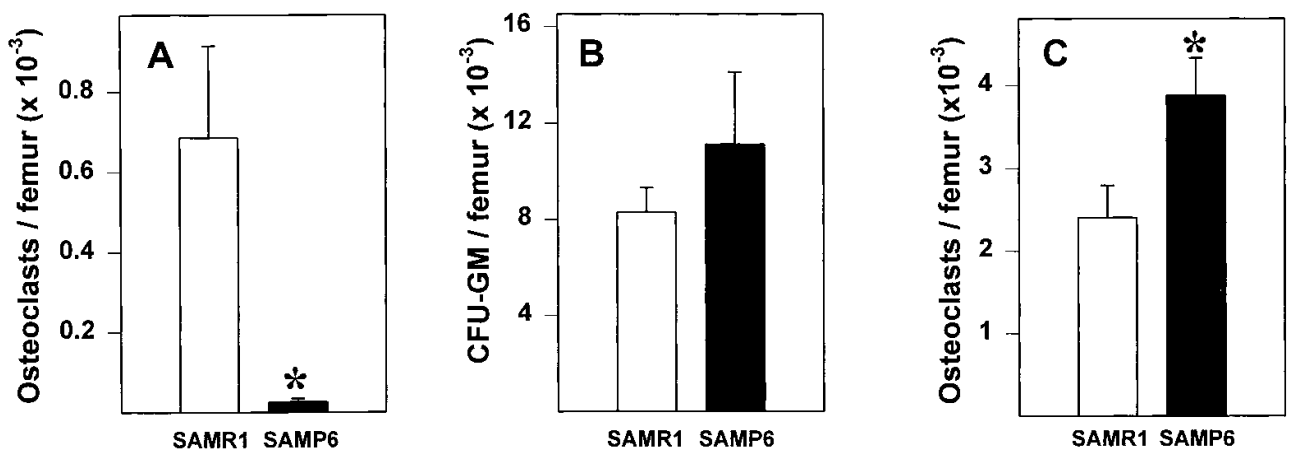

Figure 5. Osteoclast formation by marrow cells from SAMR1 and SAMP6 mice. Marrow cells from 3-4-mo-old female SAMR1 or SAMP6 mice $(n=5$ per group) were cultured alone and analyzed for osteoclast formation $(A)$, analyzed for their content of CFU-GM $(B)$, or cocultured with calvaria cells and analyzed for osteoclast formation $(C)$, as described in Methods. Cells from each animal were cultured separately in each as-

say. The data in $A$ and $C$ are derived from the same experiment. The data in $B$ are from a separate experiment. Bars represent the mean ( \pm SD) number of osteoclasts or CFU-GM colonies per femur, calculated from the total number of cells obtained from the femur of each mouse. * $P<$ 0.05 vs. SAMR1.

\section{Discussion}

Bone loss occurs when the amount of bone formed during each remodeling cycle does not exactly replace the bone removed during the preceding resorption phase. Theoretically, this could arise from $(a)$ a reduction in the number of osteoblasts that differentiate from progenitors in the marrow; $(b)$ a defect in the processes that cause the newly recruited team of differentiated osteoblasts to arrive at the remodeling site at the correct place and time; or (c) an increase in bone resorption. The evidence presented in this report demonstrates that in SAMP6 mice the first scenario is responsible for a reduction in the rate of bone formation in remodeling trabecular bone, and the reduced bone mass. Thus, at $\sim 1$ mo of age, SAMR 1 and SAMP6 mice have identical numbers of osteoblast progenitors in the marrow, identical rates of bone formation in remodeling trabecular bone, and identical BMD. However, by 3-4 mo of age, the number of osteoblast progenitors in the marrow of SAMP6 mice is reduced, and this is accompanied by a reduction in the rate of bone formation and diminished bone mass. Furthermore, a close relationship between osteoblastogenesis and both bone mass and formation is strongly suggested by the direct correlations between CFU-OB and spinal cancellous bone area, mineralizing perimeter, and mineral appositional rate. These observations are thus consistent with the accelerated aging that characterize these mice and establish that the osteopenic phenotype becomes apparent after sexual maturity.

It is important to note that the bone formation we measured in the secondary spongiosa of both growing juvenile mice and adult mice is due to remodeling activity. Indeed, we noted discrete erosion bays, as well as sites of bone formation associated with reversal lines in the cancellous bone we examined. Matsushita et al. (22) have reported previously that lon-
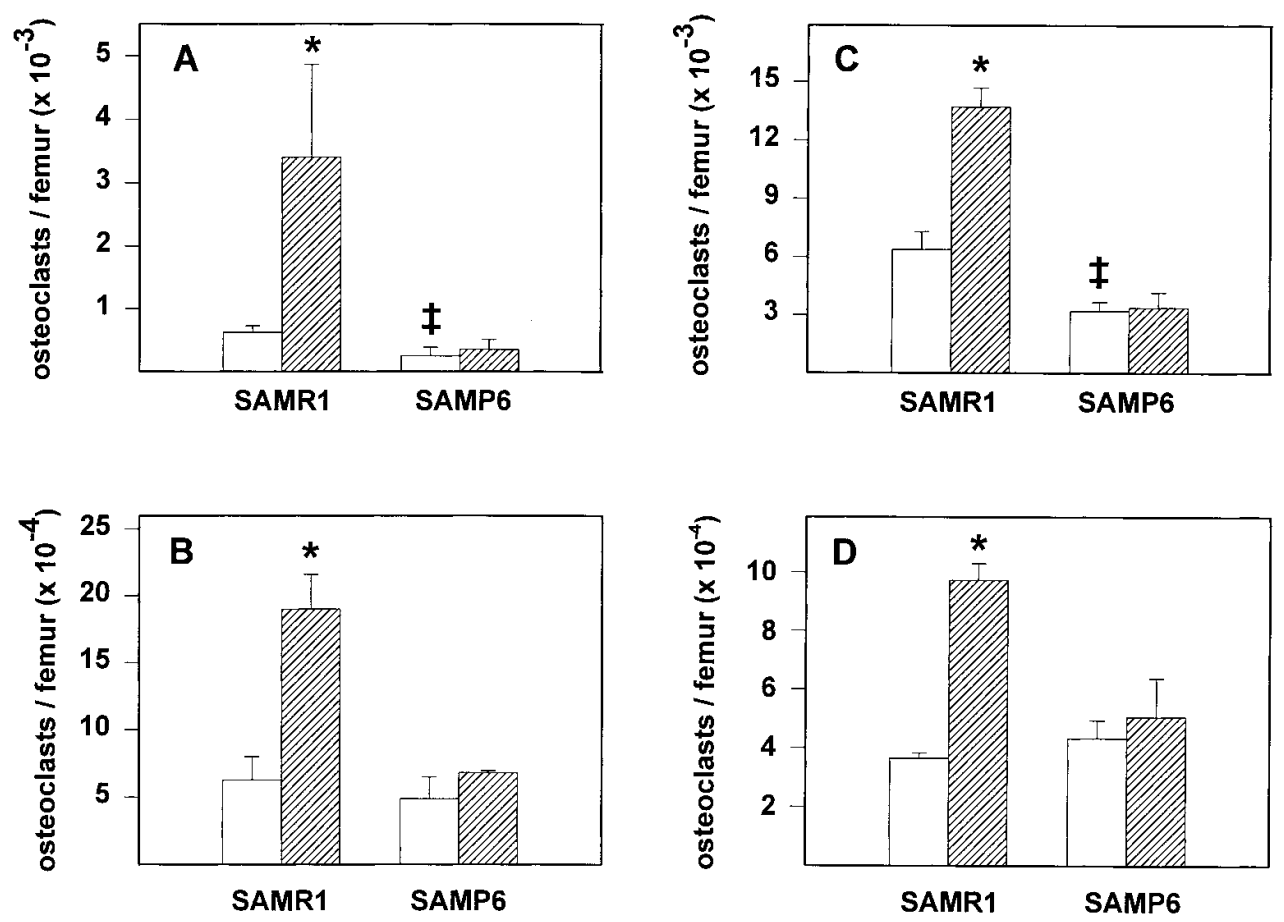

Figure 6. Effect of sex steroid deficiency on osteoclastogenesis in SAMR1 and SAMP6 mice. Female SAMR1 and SAMP6 mice were sham-operated (open bars) or ovariectomized (striped bars) ( $A$ and $B$ ), and male SAMR1 and SAMP6 mice were sham-operated (open bars) or orchidectomized (striped bars) $(C$ and $D)$. At the time of operation, mice were $3-4$ mo old $(n=$ 4-5 per group). 4 wk after the operation, bone marrow cells were obtained from the femur and cultured alone ( $A$ and $C$ ), or cocultured with neonatal calvaria cells ( $B$ and $D)$, and analyzed for osteoclast formation as described in Methods. Cells from each animal were cultured separately. Bars represent the mean $( \pm \mathrm{SD})$ number of osteoclasts per femur, calculated from the total number of cells obtained from each femur. $* P<0.05$ vs. sham-operated mice; ${ }^{\ddagger} P<0.05$ vs. sham-operated SAMR1. 
gitudinal growth of the femur and the age at which peak bone mass is attained are identical in SAMR1 and SAMP6 mice. Thus, at this stage, there is no evidence that alterations in growth or modeling in bone of SAMP6 mice contribute to the development of their osteopenia.

The osteopenic phenotype of SAMP6 was originally noted by the occurrence of "spontaneous" tibial fractures. Additional studies revealed a reduction in the cortical thickness of the diaphysis of the femur that became apparent at 3-4 mo of age (22). Although our histomorphometric measurements revealed significant differences in bone architecture, cell number, and the kinetics of bone formation in the cancellous bone of SAMR1 and SAMP6 mice, this approach can provide only limited information on overall bone mass due to the small size of the bone specimen available for study and the microheterogeneity of cancellous bone. Thus, although a significant reduction in cancellous bone was apparent in vertebral bone, the reduction in the distal femur was less impressive. However, a significant reduction in trabecular width was noted in the distal femur, indicating that the decreased number of osteoblast progenitors at this site was accompanied by trabecular bone atrophy. With further remodeling of this bone in the face of insufficient osteoblasts, additional bone loss will ensue.

Using a Hologic QDR-2000 Plus densitometer adapted for the measurement of bone mass by DEXA in live mice, we noted a reduction in bone mass in vertebral bone of SAMP6 mice at 3-4 mo of age. In addition, we observed that very old (15 mo) animals exhibited osteopenia in all skeletal sites examined. Thus, SAMP6 mice exhibit progressive loss of bone between 3 and 15 mo of age, a phenomenon which apparently did not occur in SAMR1 mice. However, as they approach 15 mo of age, a larger percentage of SAMP6 mice die, creating a group of unique survivors. Therefore, additional longitudinal studies are required to fully characterize age-related bone loss in these mice. In any event, the development of DEXA methodology for use in live mice, described here for the first time, makes such studies feasible and permits concurrent histomorphometric and BMD determinations. We believe that this methodologic advance will also allow exploitation of the wealth of information on the genetics and strain diversity in the mouse for studies on the development of osteopenia (35).

The marrow of adult SAMP6 mice has few osteoblast progenitors, but normal numbers of mulitpotential mesenchymal progenitors, CFU-F. Analysis of the properties of individual CFU-F colonies formed in ex vivo marrow of cultures indicated a considerable degree of heterogeneity with respect to the number of cells in each colony and the level of alkaline phosphatase expression, reflecting differences in the properties of the progenitors that gave rise to each colony. Comparison of individual colonies formed in cultures from SAMP6 and SAMR1 mice indicated that the mesenchymal progenitors of SAMP6 mice gave rise to smaller colonies than marrow of SAMR1 mice. These findings suggest that the marrow of SAMP6 mice is deficient in a population of rapidly dividing progenitors committed to the osteoblast lineage. Previous studies have indicated increased adipogenesis in marrow of SAMP6 mice (36). In addition, we have reported elsewhere evidence for an increase in hematopoietic activity in marrow of adult SAMP6 mice (37). These findings are consistent with the notion that there is an alteration in the pattern of differentiation of the progenitors of the mesenchymal cell lineage in the bone marrow of SAMP6 mice.
Because we found decreased osteoblast progenitors and reduced bone formation rate in bone of adult SAMP6 mice, we expected that the number of osteoblasts in bone would also be reduced. Although the mean number of osteoblasts was lower in cancellous bone of SAMP6 mice, the reduction was not statistically significant. This result may be due to high variation in histomorphometric measurements of osteoblasts, as noted previously (38). Compared with osteoblasts of rat or human bone, murine osteoblasts exhibited reduced size, were columnar rather than cuboidal, and exhibited less granularity. Due to their less distinctive features, therefore, we may have overestimated the number of mature osteoblasts that are synthesizing bone matrix using current histological criteria. Some of these cells could have been osteoblast progenitors or inactive osteoblasts. Development of new methods to identify matrix-synthesizing osteoblasts should allow more precise quantification of these cells.

The cancellous bone of adult SAMP6 mice exhibited a dramatic reduction in the number of osteoclasts, compared with bone of SAMR1 mice. Further, the marrow of SAMP6 mice, in contrast to the control SAMR1 mice, did not exhibit increased osteoclastogenesis when challenged by the removal of sex steroids. This phenomenon was due to a defect in the ability of stromal/osteoblastic cells in the marrow SAMP6 mice to support osteoclast formation and therefore was secondary to the defective osteoblastogenesis. In view of the extensive evidence that cells of the stromal/osteoblastic cell lineage are required for osteoclast formation, this finding was not unexpected. However, this is the first in vivo demonstration of this dependency. More important, the evidence that loss of sex steroids failed to increase osteoclastogenesis in the marrow of SAMP6 mice demonstrates the essential role of stromal/osteoblastic cells in the increased osteoclastogenesis caused by loss of sex steroids. In fact, this observation is consistent with the view that cells of the stromal/osteoblastic cell lineage are the principal targets of estrogens and androgens and therefore the essential mediators of the increased osteoclastogenesis caused by sex steroid deficiency $(24,25)$.

The trabecular bone of SAMP6 mice exhibits many features that are analogous to that of aging humans. In addition to osteopenia, the trabeculae were infrequent and widely separated in the spine, suggesting increased perforation by osteoclasts. Although osteoclast numbers were reduced in SAMP6 mice, the rate of bone turnover was still sufficiently rapid to explain the osteopenia in these mice. Based on the bone turnover measured at 3 mo of age, $0.5-4 \%$ of the cancellous bone is remodeled every $10 \mathrm{~d}$. Thus, the osteoblast defect in SAMP6 was severe enough to preempt the bone formation required to reconstitute the previously eroded cavities. The reduced remodeling and the altered architecture of bone of SAMP6 mice are cardinal features of bone in aging humans. Thus, many cellular and architectural features of aging bone in humans are duplicated in this mouse model in a way that permits investigation of the cause of this osteopenia in animals that are the same age. Hence, the SAMP6 model should be useful for the exploration of the molecular and cellular basis of the relationship among osteoblastogenesis, bone formation in remodeling bone, and bone mass in a context that is similar to the agerelated osteopenia that occurs in humans.

In conclusion, the demonstration of a close association between the development of a defect in osteoblastogenesis in a murine model of accelerated aging with resulting decreases in 
osteoclast formation, bone formation, and bone mass strongly suggests the critical role of the bone marrow in the development of the osteopenia in this model. Because a decreased supply of osteoblasts may explain the diminished amount of bone formed during each remodeling cycle during aging in humans, our findings establish the validity of the SAMP6 mouse as an animal model of involutional osteoporosis. Finally, they pave the way for chromosome mapping studies aiming to elucidate genes that contribute to osteopenia (35).

\section{Acknowledgments}

The authors thank Dianne Lindsey, Frances Miller, Randal Shelton, Catherine Smith, and Patty White for their valuable technical assistance in the conduct of this work.

This work was supported by National Institutes of Health grant AR-41313 and by the Department of Veterans Affairs.

\section{References}

1. Parfitt, A.M. 1992. The two-stage concept of bone loss revisited. Triangle. 31:99-110.

2. Parfitt, A.M. 1990. Bone-forming cells in clinical conditions. In Bone. Volume 1. The Osteoblast and Osteocyte. B.K. Hall, editor. Telford Press and CRC Press, Boca Raton, FL. 351-429.

3. Parfitt, A.M., C.H.E. Mathews, A.R. Villanueva, M. Kleerekoper, B. Frame, and D.S. Rao. 1983. Relationships between surface, volume, and thickness of iliac trabecular bone in aging and in osteoporosis. Implications for the microanatomic and cellular mechanism of bone loss. J. Clin. Invest. 72:13961409.

4. Eriksen, E.F., S.F. Hodgson, R. Eastell, S.L. Cedel, W.M. O'Fallon, and B.L. Riggs. 1990. Cancellous bone remodeling in type I (postmenopausal) osteoporosis: quantitative assessment of rates of formation, resorption, and bone loss at tissue and cellular levels. J. Bone Miner. Res. 5:311-319.

5. Balena, R., B.C. Toolan, M. Shea, A. Markatos, E.R. Myers, S.C. Lee, E.E. Opas, J.G. Seedor, H. Klein, D. Frankenfield, et al. 1993. The effects of 2-year treatment with the aminobisphosphonate alendronate on bone metabolism, bone histomorphometry, and bone strength in ovariectomized nonhuman primates. J. Clin. Invest. 92:2577-2586.

6. Poli, V., R. Balena, E. Fattori, A. Markatos, A. Yamamoto, H. Tanaka, G. Ciliberto, G.A. Rodan, and F. Costantini. 1994. Interleukin-6 deficient mice are protected from bone loss caused by estrogen depletion. EMBO (Eur. Mol. Biol. Organ.) J. 13:1189-1196.

7. Gallagher, J.C., D. Goldgar, and A. Moy. 1987. Total bone calcium in normal women: effect of age and menopause status. J. Bone Miner. Res. 2:491496.

8. Heaney, R.P. 1990. Estrogen-calcium interactions in the postmenopause: a quantitative description. Bone Miner. 11:67-84.

9. Nordin, B.E.C., A.G. Need, A. Bridges, and M. Horowitz. 1992. Relative contributions of years since menopause, age, and weight to vertebral density in postmenopausal women. J. Clin. Endocrinol. \& Metab. 74:20-23.

10. Eastell, R., A.L. Yergey, N.E. Vieira, S.L. Cedel, R. Kumar, and B.L. Riggs. 1991. Interrelationship among vitamin D metabolism, true calcium absorption, parathyroid function, and age in women: evidence of an age-related intestinal resistance to 1,25-dihydroxyvitamin D action. J. Bone Miner. Res. 6: $125-132$

11. Sherman, S.S., J.D. Tobin, B.W. Hollis, C.M. Gundberg, T.A. Roy, and C.C. Plato. 1992. Biochemical parameters associated with low bone density in healthy men and women. J. Bone Miner. Res. 7:1123-1130.

12. Tsai, K.S., H.W. Wahner, K.P. Offord, L.J. Melton III, R. Kumar, and B.L. Riggs. 1987. Effect of aging on vitamin D stores and bone density in women. Calcif. Tissue Int. 40:241-243.

13. McKenna, M.J. 1992. Differences in vitamin D status between countries in young adults and the elderly. Am. J. Med. 93:69-77.

14. Lindsay, R., D.M. Hart, C. Forrest, and C. Baird. 1980. Prevention of spinal osteoporosis in oophorectomised women. Lancet. 2:1151-1154.

15. Orwoll, E.S., and R.F. Klein. 1995. Osteoporosis in men. Endocr. Rev. 16:87-116.

16. Lips, P., P. Courpron, and P.J. Meunier. 1978. Mean wall thickness of trabecular bone packets in the human iliac crest: changes with age. Calcif. Tissue Res. 26:13-17.

17. Parfitt, A.M., A.R. Villanueva, J. Foldes, and D.S. Rao. 1995. Relations between histologic indices of bone formation: implications for the pathogenesis of spinal osteoporosis. J. Bone Miner. Res. 10:466-473.

18. Parisien, M., R.W.E. Mellish, S.J. Silverberg, E. Shane, R. Lindsay, J.P. Bilezikian, and D.W. Dempster. 1992. Maintenance of cancellous bone connectivity in primary hyperparathyroidism: trabecular strut analysis. J. Bone Miner. Res. 7:913-919.

19. Steiniche, T., C. Hasling, P. Charles, E.F. Eriksen, L. Mosekilde, and F. Melsen. 1989. A randomized study on the effects of estrogen/gestagen or high dose oral calcium on trabecular bone remodeling in postmenopausal osteoporosis. Bone. 10:313-320.

20. Manolagas, S.C., and R.L. Jilka. 1995. Bone marrow cytokines and bone remodeling. Emerging insights into the pathophysiology of osteoporosis. $N$. Engl. J. Med. 332:305-311

21. Takeda, T., M. Hosokawa, K. Higuchi, M. Hosono, I. Akiguchi, and H. Katoh. 1994. A novel murine model of aging, senescence-accelerated mouse (SAM). Arch. Gerontol. Geriatr. 19:185-192.

22. Matsushita, M., T. Tsuboyama, R. Kasai, H. Okumura, T. Yamamuro, K. Higuchi, A. Kohno, T. Yonezu, and A. Utani. 1986. Age-related changes in bone mass in the senescence-accelerated mouse (SAM). SAM-R/3 and SAM-P/6 as new murine models for senile osteoporosis. Am. J. Pathol. 125:276-283.

23. Kitado, H., K. Higuchi, and T. Takeda. 1994. Molecular genetic characterization of the senescence-accelerated mouse (SAM) strains. J. Gerontol. 49: B247-B254

24. Jilka, R.L., G. Hangoc, G. Girasole, G. Passeri, D.C. Williams, J.S Abrams, B. Boyce, H. Broxmeyer, and S.C. Manolagas. 1992. Increased osteoclast development after estrogen loss: mediation by interleukin-6. Science (Wash. DC). 257:88-91.

25. Bellido, T., R.L. Jilka, B.F. Boyce, G. Girasole, H. Broxmeyer, S.A Dalrymple, R. Murray, and S.C. Manolagas. 1995. Regulation of interleukin-6, osteoclastogenesis and bone mass by androgens: the role of the androgen receptor. J. Clin. Invest. 95:2886-2895.

26. Owen, M. 1985. Lineage of osteogenic cells and their relationship to the stromal system. In Bone and Mineral Research. Vol. III. W.A. Peck, editor. Elsevier Science Publishers, Amsterdam. 1-25.

27. Falla, N., P. Van Vlasselaer, J. Bierkens, B. Borremans, G. Schoeters, and U. Van Gorp. 1993. Characterization of a 5-fluorouracil-enriched osteoprogenitor population of the murine bone marrow. Blood. 82:3580-3591.

28. Korzeniewski, C., and D.M. Callewaert. 1983. An enzyme-release assay for natural cytotoxicity. J. Immunol. Methods. 64:313-320.

29. Weinstein, R.S., J.L. Underwood, M.S. Hutson, and H.F. DeLuca. 1984 Bone histomorphometry in vitamin D-deficient rats infused with calcium and phosphorus. Am. J. Physiol. 246:E499-E505.

30. Weinstein, R.S., and N.H. Bell. 1988. Diminished rates of bone formation in normal black adults. N. Engl. J. Med. 319:1698-1701.

31. Parfitt, A.M., M.K. Drezner, F.H. Glorieux, J.A. Kanis, H. Malluche, P.J. Meunier, S.M. Ott, and R.R. Recker. 1987. Bone histomorphometry: standardization of nomenclature, symbols, and units. Report of the ASBMR Histomorphometry Nomenclature Committee. J. Bone Miner. Res. 2:595-610.

32. Girasole, G., R.L. Jilka, G. Passeri, S. Boswell, G. Boder, D.C. Williams, and S.C. Manolagas. 1992. 17ß-estradiol inhibits interleukin-6 production by bone marrow-derived stromal cells and osteoblasts in vitro. A potential mechanism for the antiosteoporotic effect of estrogens. J. Clin. Invest. 89:883-891.

33. Girasole, G., G. Passeri, R.L. Jilka, and S.C. Manolagas. 1994. Interleukin-11: a new cytokine critical for osteoclast development. J. Clin. Invest. 93: 1516-1524.

34. Takahashi, N., T. Akatsu, N. Udagawa, T. Sasaki, A. Yamaguchi, J.M. Moseley, T.J. Martin, and T. Suda. 1988. Osteoblastic cells are involved in osteoclast formation. Endocrinology. 123:2600-2602.

35. Shmookler Reis, R.J., R.S. Weinstein, K. Takahashi, R.L. Jilka, and S.C. Manolagas. 1995. A strategy for chromosome mapping of osteopenia-associated genes in the senescence accelerated mouse (SAM): use of high-resolution dual-energy x-ray absorptionmetry. J. Bone Miner. Res. 10(Suppl. 1):S161a. (Abstr.)

36. Uchiyama, Y., K. Miyama, T. Katagiri, A. Yamaguchi, H. Takamori, K. Nakashima, T. Sato, and T. Suda. 1994. Adipose conversion is accelerated in bone marrow cells of congenitally osteoporotic SAMP6 mice. J. Bone. Miner. Res. 9 (Suppl. 1):S321a. (Abstr.)

37. Kajkenova, O, I Gubrij, S.P. Hauser, K. Takahashi, R.L Jilka, S.C. Manolagas, and D.A. Lipschitz. 1995. Increased hematopoiesis accompanies reduced osteoblastogenesis in the senescence-accelerated mouse (SAM-P/6). $J$. Bone Miner. Res. 10(Suppl. 1):S431a. (Abstr.)

38. Weinstein, R.S. 1982. Decreased mineralization in hemodialysis patients after subtotal parathyroidectomy. Calcif. Tissue Int. 34:16-20. 